\title{
Release of $\alpha_{2}$-Plasmin Inhibitor from Plasma Fibrin Clots by Activated Coagulation Factor XIII

\author{
Its Effect on Fibrinolysis
}

\author{
Jun Mimuro, Shigeru Kimura, and Nobuo Aoki \\ Institute of Hematology and Department of Medicine, Jichi Medical School, Tochigi-Ken 329-04, Japan
}

\begin{abstract}
When blood coagulation takes place in the presence of calcium ions, $\alpha_{2}$-plasmin inhibitor $\left(\alpha_{2} P I\right)$ is cross-linked to fibrin by activated coagulation Factor XIII (XIIIa) and thereby contributes to the resistance of fibrin to fibrinolysis. It was previously shown that the cross-linking reaction is a reversible one, since the $\alpha_{2} \mathrm{PI}$ fibrinogen cross-linked complex could be dissociated. In the present study we have shown that the $\alpha_{2}$ PI-fibrin cross-linking reaction is also a reversible reaction and $\alpha_{2} \mathrm{PI}$ which had been cross-linked to fibrin can be released from fibrin by disrupting the equilibrium, resulting in a decrease of its resistance to fibrinolysis. When the fibrin clot formed from normal plasma in the presence of calcium ions was suspended in $\alpha_{2}$ PI-deficient plasma of buffered saline, $\alpha_{2} P I$ was gradually released from fibrin on incubation. When $\alpha_{2}$ PI was present in the suspending milieu, the release was decreased inversely to the concentrations of $\alpha_{2} P I$ in the suspending milieu. The release was accelerated by supplementing XIIIa or the presence of a high concentration of the $\mathrm{NH}_{2}$-terminal 12-residue peptide of $\alpha_{2} \mathrm{PI}$ ( $\mathrm{N}$-peptide) which is cross-linked to fibrin in exchange for the release of $\alpha_{2} P I$. When the release of $\alpha_{2}$ PI from fibrin was accelerated by XIIIa or $\mathbf{N}$ peptide, the fibrin became less resistant to the fibrinolytic process, resulting in an acceleration of fibrinolysis which was proportional to the degree of the release of $\alpha_{2}$ PI. These results suggest the possiblity that $\alpha_{2} P I$ could be released from fibrin in vivo by disrupting the equilibrium of the $\alpha_{2}$ PI-fibrin cross-linking reaction, and that the release would result in accelerated thrombolysis.
\end{abstract}

\section{Introduction}

When blood coagulation takes place $\sim 20 \%$ of the $\alpha_{2}$-plasmin inhibitor $\left(\alpha_{2} \mathrm{PI}\right){ }^{1}$ a major inhibitor of fibrinolysis (1) present in plasma, is cross-linked to the $\alpha$-chain of fibrin by plasma trans-

This work was presented in part at the International Congress on Fibrinolysis, 29 March 1984.

Dr. Aoki's present address is First Department of Medicine, Tokyo Medical and Dental University, Yushima 1-5-45, Bunkyo-Ku, Tokyo 113, Japan.

Received for publication 27 August 1984 and in revised form 28 October 1985

1. Abbreviations used in this paper: $\alpha_{2} \mathrm{PI}, \alpha_{2}$-plasmin inhibitor; ${ }^{125} \mathrm{I}-\mathrm{FDP}$, radiolabeled fibrin degradation products; $\mathrm{N}$-peptide, the $\mathrm{NH}_{2}$-terminal 12-residue peptide of $\alpha_{2} \mathrm{PI}$; t-PA, tissue plasminogen activator; XIIIa, activated coagulation Factor XIII.

J. Clin. Invest.

(C) The American Society for Clinical Investigation, Inc.

0021-9738/86/03/1006/08 \$1.00

Volume 77, March 1986, 1006-1013 glutaminase, activated blood coagulation Factor XIII (XIIIa) (2-4). The $\alpha_{2}$ PI thus cross-linked renders the fibrin clot more resistant to fibrinolysis. Particularly, the naturally occurring fibrinolytic process that occurs subsequently to fibrin formation and is caused by fibrin-associated plasminogen activation (5) is mainly inhibited by $\alpha_{2}$ PI cross-linked to fibrin (6).

$\alpha_{2}$ PI serves only as a glutamine substrate for XIIIa in the cross-linking reaction (4), and the cross-linking occurs between lysine residues of fibrin $\alpha$-chains and a glutamine residue of the $\alpha_{2} \mathrm{PI}$ molecule that is the second residue from the $\mathrm{NH}_{2}$-terminal (4).

We have previously suggested that the $\alpha_{2}$ PI cross-linking reaction may be a reversible equilibrium reaction that is common to the general cross-linking reaction catalyzed by transglutaminases $(7,8)$, since the $\alpha_{2}$ PI-fibrinogen cross-linked complex could be dissociated to each of its components to reach a new equilibrium state when the isolated complex was incubated with XIIIa (9). It is important to know if the $\alpha_{2}$ PI cross-linked with fibrin could be released physiologically, because such a release of $\alpha_{2}$ PI from fibrin would make the fibrin less resistant to the fibrinolytic process, thereby accelerating fibrinolysis.

In the present study we have demonstrated that $\alpha_{2}$ PI once cross-linked with fibrin could be released by the function of XIIIa and the release could result in an acceleration of fibrinolysis.

\section{Methods}

Plasma. Blood was withdrawn from a normal individual, a patient with congenital deficiency of $\alpha_{2} \mathrm{PI}$ (10), or a patient with congenital deficiency of Factor XIII, into 0.1 vol of $3.8 \%$ sodium citrate or into 0.1 vol of 100 $\mathrm{U} / \mathrm{ml}$ heparin. The blood was then centrifuged to prepare platelet-rich or -poor plasma. The concentration of $\alpha_{2} \mathrm{PI}$ in plasma was immunologically determined (2).

Purified proteins. $\alpha_{2} \mathrm{PI}$ was purified from human plasma by the previously described method (11). The concentration was determined spectrophotometrically using $\mathrm{E}_{\mathrm{lcm}}^{1 \%}=7.03$ at $280 \mathrm{~nm}$ (11). The protein was dissolved in Tris-buffered saline $(0.05 \mathrm{M}$ Tris- $\mathrm{HCl}, 0.15 \mathrm{M} \mathrm{NaCl}, \mathrm{pH}$ 7.4). Human fraction $I-4$, prepared according to the method of Blombäck and Blombäck (12), was used as the fibrinogen preparation after removing contaminating plasminogen and plasma fibronectin from the preparation with lysine-Sepharose (13) and gelatin-Sepharose (14), respectively. The concentration was determined spectrophotometrically using $\mathrm{E}_{\mathrm{lcm}}^{\mathrm{l} \%}=15.1$ at $280 \mathrm{~nm}$ (12) and the coagulable protein was $>95 \%$. Factor XIII was purified from human plasma by the method described by Curtis and Lorand (15). Factor XIII activity was assayed by the dansylcadaverine incorporation method as described by Lorand et al. (16). $1 \mathrm{U}$ of Factor XIII is defined as the activity of Factor XIII present in $1 \mathrm{ml}$ normal pooled standard plasma. Thrombin-modified Factor XIII was prepared as described previously (4) and was converted to XIIIa by calcium ions added at the time of the experiments. Purified thrombin was prepared from a bovine thrombin preparation (Mochida Pharmaceuticals, Tokyo, Japan) according to the method of Lundblad (17). The hirudin (a thrombin inhibitor obtained from leeches) used was a grade IV preparation from the Sigma Chemical Co., St. Louis, MO, and was shown to have 
no plasmin inhibitor activity when tested by the chromogenic (18) and clot lysis (19) methods. Tissue plasminogen activator (t-PA) was purified from the culture media of a human melanoma cell line according to the method of Rijken and Collen (20). The t-PA activity was assayed by the clot lysis method using a calibration curve constructed with the WHO standard preparation kindly supplied by Dr. P. J. Gaffney, National Institute for Biological Standards and Control, London, England. Native Glu-plasminogen, whose amino-terminal group is glutamic acid, was prepared from fresh plasma in the presence of aprotinin (10 kallikrein inhibitor units/ml) (Mochida Pharmaceuticals) by affinity chromatography with lysine-Sepharose followed by DEAE-Sephadex chromatography (21). Plasmin was prepared by activating plasminogen with urokinase-coupled Sepharose 4B (11). Plasmin activity was determined by the amidolytic method (22) using the chromogenic substrate, H-D-ValLeu-Lys-p-nitroanilide (S-2251; Daiichi Pure Chemicals Co., Tokyo, Japan).

$\mathrm{NH}_{2}$-terminal peptide of $\alpha_{2} P I$. The $\mathrm{NH}_{2}$-terminal 12-residue peptide of $\alpha_{2}$ PI (N-peptide), Asn-Gln-Glu-Gln-Val-Ser-Pro-Leu-Thr-Gly-LeuLys- $\mathrm{NH}_{2}$. AcOH was synthesized and kindly supplied by Dr. H. Tani, Tokyo Research Institute, Kowa Co., Tokyo, Japan.

Radioiodination of protein. $\alpha_{2} \mathrm{PI}$ and fibrinogen were radioiodinated by solid-state lactoperoxidase-glucose oxidase (Enzymobead; Bio-Rad Laboratories, Richmond, $\mathrm{CA}$ ) and $\mathrm{Na}^{125} \mathrm{I}(17 \mathrm{Ci} / \mathrm{mg})$ (New England $\mathrm{Nu}$ clear, Boston, MA). Free unconjugated radiolabeled compounds were removed by gel filtration using Sephadex G-10. The labeled $\alpha_{2} \mathrm{PI}$ and fibrinogen preparations had specific activities of $1 \times 10^{6} \mathrm{cpm} / \mu \mathrm{g}$ and 1.2 $\times 10^{6} \mathrm{cpm} / \mu \mathrm{g}$, respectively.

Release of $\alpha_{2}$ PI from fibrin clots. 100 or $40 \mu \mathrm{l}$ of normal citrated plasma containing a trace amount of radiolabeled $\alpha_{2} \mathrm{PI}\left(7.5 \times 10^{4} \mathrm{cpm} /\right.$ $\mathrm{ml}$ plasma) and aprotinin ( $10 \mathrm{U} / \mathrm{ml}$ plasma) was clotted with calcium chloride $(25 \mathrm{mM})$ and thrombin $(1 \mathrm{U} / \mathrm{ml})$. The clotted plasma was incubated at $37^{\circ} \mathrm{C}$ for $30 \mathrm{~min}$. Aprotinin was used to prevent fibrinolysis from occurring during the incubation. The clot was subsequently squeezed and washed three times by soaking and shaking it for 5 min each time in $400 \mu$ l of Tris-buffered saline $(0.05 \mathrm{M}$ Tris- $\mathrm{HCl} / 0.15 \mathrm{M} \mathrm{NaCl}, \mathrm{pH}$ 7.4) containing $0.2 \%$ human albumin (Miles Ames Div., Miles Laboratories Inc., Elkhart, IN). The washed clot was then suspended in 500 $\mu \mathrm{l}$ of normal or $\alpha_{2} \mathrm{PI}$-deficient heparinized plasma or buffered saline and incubated at $37^{\circ} \mathrm{C}$. The suspending plasma contained aprotinin $(10 \mathrm{U} /$ $\mathrm{ml})$ and hirudin $(10 \mathrm{U} / \mathrm{ml})$ (Sigma Chemical Co.). The suspending buffered saline contained XIIIa $(0-0.6 \mathrm{U} / \mathrm{ml})$ and aprotinin $(10 \mathrm{U} / \mathrm{ml})$. As control experiments, EDTA $(2 \mathrm{mM})$ and iodoacetamide $(1 \mathrm{mM})$ were further included in the suspending media to inhibit XIIIa activity. During the incubation, aliquots of $20 \mu \mathrm{l}$ were removed from the suspending media at intervals for radioactive counting. Results were expressed as the percent release of radiolabel from the clot, which was calculated from the counts, applying a correction for the influence of repeated subsampling on the volume. In some experiments, the release was also expressed as moles. The experiments were carried out in triplicate.

To confirm the cross-linking of $\alpha_{2} \mathrm{PI}$ to fibrin and its subsequent release, the washed clot, together with buffered saline suspending the clot, was lyophilized as a whole before and after the incubation, then solubilized and subjected to sodium dodecyl sulfate (SDS)-polyacrylamide gel electrophoresis. The washed clot was prepared from $100 \mu$ l of normal citrated plasma or $100 \mu \mathrm{l}$ of fibrinogen $(2.3 \mathrm{mg} / \mathrm{ml})$ in Tris-buffered saline containing $\alpha_{2} \mathrm{PI}(30 \mu \mathrm{g} / \mathrm{ml})$ and Factor XIII $(0.2 \mathrm{U} / \mathrm{ml})$, but otherwise all the other conditions were the same as described above. The solubilization was carried out by incubation for $20 \mathrm{~min}$ at $100^{\circ} \mathrm{C}$ with $40 \mu \mathrm{l}$ of $10 \%$ SDS, $4 \mathrm{M}$ urea, $10 \%$ mercaptoethanol. The dissolved samples were subjected to the electrophoresis on a slab gel or disc gel according to the procedures of Laemmli (23) using a 7.5\% separating gel and a $4 \%$ stacking gel. High molecular weight standards (Bio-Rad Laboratories) were run in parallel. After the electrophoresis, the gels were stained with Coomassie Brilliant Blue and destained by diffusion. The $\alpha_{2} \mathrm{PI}$ was identified by radioautography by exposing the dried slab gel to X-ray film (Lo-dose film; E. I. Du Pont de Nemours \& Co., Wilmington, DE) in the presence of intensifying screens (Lo-dose/2 mammography intensi- fying screen; E. I. Du Pont de Nemours \& Co.) for $24 \mathrm{~h}$ at $-70^{\circ} \mathrm{C}$. The $\alpha_{2} \mathrm{PI}$ was also identified by cutting the disc gel transversely into slices of $3 \mathrm{~mm}$ thickness and counting each slice for radioactivity.

$\alpha_{2} \mathrm{PI}$ released was examined for its antiplasmin activity. For that purpose, $2 \mathrm{ml}$ of normal plasma containing radiolabeled $\alpha_{2} \mathrm{PI}$ was clotted, washed, suspended, and incubated in $2 \mathrm{ml}$ of buffered saline containing $0.6 \mathrm{U} / \mathrm{ml}$ XIIIa in the same way as described above, except for the omission of aprotinin. At 20 and $60 \mathrm{~min}$ of incubation, aliquots of 800 or 2 $\mu \mathrm{l}$ were removed from the suspending media for antiplasmin activity or radioactive counting, respectively. For antiplasmin activity an aliquot of $800 \mu \mathrm{l}$ was mixed at $37^{\circ} \mathrm{C}$ with $200 \mu \mathrm{l}$ of $5 \mathrm{mM} \mathrm{S}-2251$ and then with $100 \mu \mathrm{l}$ of plasmin solution ( $4 \mathrm{nmol}$ of substrate hydrolyzed/second per $\mathrm{ml}$ ). Increase of absorbance at $405 \mathrm{~nm}$ was monitored by a DU-8 spectrophotometer (Beckman Instruments, Inc., Fullerton, CA), and the immediate-type antiplasmin activity was calculated as described previously (18) using a standard curve constructed with normal plasma and expressed as a percent of the standard normal plasma.

Incorporation of $\alpha_{2} P I$ to clot. The washed clot prepared from $100 \mu \mathrm{l}$ of normal plasma was suspended and incubated at $37^{\circ} \mathrm{C}$ in $500 \mu \mathrm{l}$ of $\alpha_{2}$ PI-deficient heparinized plasma supplemented with various concentrations of $\alpha_{2}$ PI containing a trace amount of radiolabeled $\alpha_{2} \mathrm{PI}$. The suspending plasma contained aprotinin $(10 \mathrm{U} / \mathrm{ml})$ and hirudin $(10 \mathrm{U} /$ $\mathrm{ml})$. As control experiments, EDTA $(20 \mathrm{mM})$ and iodoacetamide (1 $\mathrm{mM}$ ) were further included in the suspending plasma. After various lengths of incubation, the clot was removed, washed, and counted for radioactivity. The amount of $\alpha_{2} \mathrm{PI}$ incorporated into the clot from the suspending plasma was calculated from the count of the clot and the specific radioactivity of $\alpha_{2} \mathrm{PI}$ used.

Measurement of fibrinolysis. $2 \mathrm{ml}$ of platelet-rich plasma were mixed with $10 \mu \mathrm{l}$ of radiolabeled fibrinogen. A $180 \mu \mathrm{l}$ aliquot of this mixture was mixed and incubated with $20 \mu \mathrm{l}$ of calcium chloride $(250 \mathrm{mM})$. The clot soon formed and underwent retraction. After incubation at $37^{\circ} \mathrm{C}$ for $30 \mathrm{~min}$, the clot was suspended by the addition of $800 \mu$ l of plateletpoor plasma containing t-PA $(8.4 \mathrm{U} / \mathrm{ml})$, hirudin $(1 \mathrm{U} / \mathrm{ml})$ and various concentrations of the $\mathrm{NH}_{2}$-terminal 12-residue peptide of $\alpha_{2} \mathrm{PI}$ ( $\mathrm{N}$-peptide) and was further incubated at $37^{\circ} \mathrm{C}$ for as long as $20 \mathrm{~h}$. The purpose of including hirudin in the suspending plasma milieu was to prevent the suspending plasma from being clotted by thrombin released from the clot. In another set of experiments, t-PA was added to plasma before clotting, and the clot formed was washed and suspended in buffered saline containing plasminogen. $2 \mu \mathrm{l}$ of t-PA $(4,200 \mathrm{U} / \mathrm{ml})$ was added to a $180-\mu \mathrm{l}$ aliquot of the platelet-poor plasma containing radiolabeled fibrinogen, and the plasma was clotted with $10 \mu \mathrm{l}$ of calcium chloride $(500 \mathrm{mM})$ and $10 \mu \mathrm{l}$ of thrombin $(20 \mathrm{U} / \mathrm{ml})$. After incubation for 30 min at $37^{\circ} \mathrm{C}$, the clot was first squeezed with a stick against the wall of the tube to express as much fluid as possible, washed three times by soaking it in $1 \mathrm{ml}$ of Tris-buffered saline containing $2 \%$ of albumin (Trisbuffered albumin saline) for $5 \mathrm{~min}$ at room temperature each time, and then finally suspended and incubated in $1 \mathrm{ml}$ of Tris-buffered albumin saline containing Glu-plasminogen $(0.18 \mathrm{mg} / \mathrm{ml})$, calcium chloride $(2.5$ $\mathrm{mM})$, and XIIIa (1.8 U/ml). As control experiments, EDTA (25 mM) and iodoacetamide $(1 \mathrm{mM})$ were included in addition to plasminogen in Tris-buffered albumin saline to inhibit XIIIa activity. The mixture containing the suspended clot was further incubated at $37^{\circ} \mathrm{C}$. To measure fibrinolysis, $20 \mu \mathrm{l}$ aliquots of the suspending plasma or buffered saline were removed at intervals for counting of radioactivity. Results were expressed as the percent release of radiolabel (radiolabeled fibrin degradation products, ${ }^{125}$ I-FDP), which was calculated from the counts, applying a correction for the influence of repeated subsampling upon the volume of the supernatant.

\section{Results}

Release of $\alpha_{2}$ PI from fibrin clot catalyzed by XIIIa. Normal citrated plasma containing radiolabeled $\alpha_{2} \mathrm{PI}$ was clotted by an addition of calcium chloride. $\alpha_{2}$ PI was rapidly cross-linked to fibrin by XIIIa generated during the coagulation. After $30 \mathrm{~min}$ 
incubation at $37^{\circ} \mathrm{C}$, the clot formed was squeezed and washed to remove unbound materials. The clot was then suspended and incubated in $\alpha_{2}$ PI-deficient plasma or buffered saline. Aprotinin was present during the entire procedure to inhibit fibrinolysis. Hirudin was present in the suspending plasma to prevent the plasma from being clotted by any thrombin that remained bound to fibrin.

As seen in Fig. 1, $\alpha_{2}$ PI was gradually released upon incubation from the fibrin clot into the suspending plasma or buffered saline. When EDTA and iodoacetamide were present in the suspending media to inhibit XIIIa, the release was significantly lower and very small. The degree of release of $\alpha_{2}$ PI was nearly the same whether the fibrin clot was suspended in $\alpha_{2} \mathrm{PI}$-deficient plasma or in buffered saline (Fig. 1). Similar degrees of release may have been caused by similar amounts of XIIIa operating in both experiments. XIIIa operating in both experiments without addition of XIIIa was the XIIIa that was activated during the coagulation and remained bound to fibrin in the washed fibrin clot (24). There was no supply of XIIIa from the suspending plasma because activation of Factor XIII in the suspending plasma was inhibited by the presence of hirudin. However, the release of $\alpha_{2}$ PI was accelerated when XIIIa was added to the buffered saline (Fig. 1). The acceleration was found to be dependent on the amount of XIIIa added. The release observed during the initial 1-h incubation is shown in Fig. 2.

Cross-linking and subsequent release were confirmed by SDSpolyacrylamide gel electrophoresis of the washed clots. In autoradiography of the polyacrylamide slab gels, there appeared a distinct radioactive band corresponding to free $\alpha_{2} \mathrm{PI}$ after the incubation of the washed clots for $4 \mathrm{~h}$ in buffered saline containing XIIIa (Fig. 3). Radiocounting of the sliced disc gels revealed that only a small proportion of $\alpha_{2}$ PI remained unbound and most of $\alpha_{2}$ PI were present in a form covalently bound to polymerized cross-linked fibrin $\alpha$-chains before the incubation (Fig. 4). After the incubation there was a remarkable increase of the height of the radioactive peak corresponding to free $\alpha_{2} \mathrm{PI}$ (Fig. 4). The increase of the radioactivity of free $\alpha_{2} \mathrm{PI}$ was around 25 or $22 \%$ of the total radioactivity in a purified fibrin clot system or in a plasma clot system, respectively.

The released $\alpha_{2}$ PI had the inhibitor activity similar to that of the original $\alpha_{2}$ PI. As shown in Fig. 5, the immediate-type

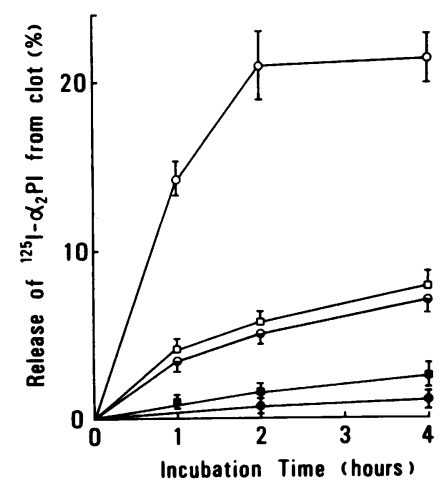

Figure 1. Release of $\alpha_{2}$ PI from fibrin clots on incubation. The washed fibrin clot prepared from normal plasma containing radiolabeled $\alpha_{2}$ PI was suspended and incubated at $37^{\circ} \mathrm{C}$ in $\alpha_{2}$ PI-deficient plasma ( $\square$ ), in buffered saline $(0)$ or in buffered saline containing XIIIa $(0.2 \mathrm{U} / \mathrm{ml})(0)$. Aprotinin was added to the plasmas and buffered saline to prevent fibrinolysis. As controls, EDTA and iodoacetamide were added to the suspending plasma $(\bullet)$ or buffered saline $(\bullet)$. After various lengths of time, cumulative release of radiolabel from the clot into the suspending medium was measured and expressed as a percentage of the total radioactivity originally present in the washed clot. Data represent the mean \pm SD of triplicate experiments. For details, see Methods.

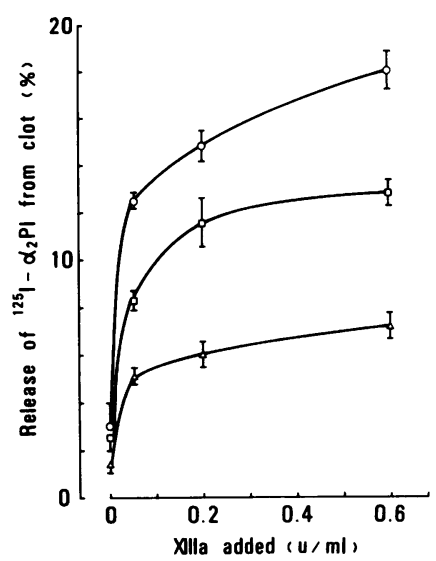

antiplasmin activity was gradually released on incubation from the washed plasma clot. The presence of iodoacetamide and EDTA suppressed the release. The release of the inhibitor activity was parallel to the release of the radioactivity; at $60 \mathrm{~min}$ incubation in Fig. 5 the total radioactivity released was also $\simeq 4 \%$ of the total radioactivity present in the original plasma.

When the clot was suspended in the medium containing $\alpha_{2} \mathrm{PI}$, some of the $\alpha_{2} \mathrm{PI}$ present in the suspending medium were incorporated into the clot by XIIIa in exchange for the release of fibrin-bound $\alpha_{2} \mathrm{PI}$. The washed clot prepared from normal plasma was suspended and incubated in the $\alpha_{2} \mathrm{PI}$-deficient plasma supplemented with various amounts of $\alpha_{2} \mathrm{PI}$. The release and

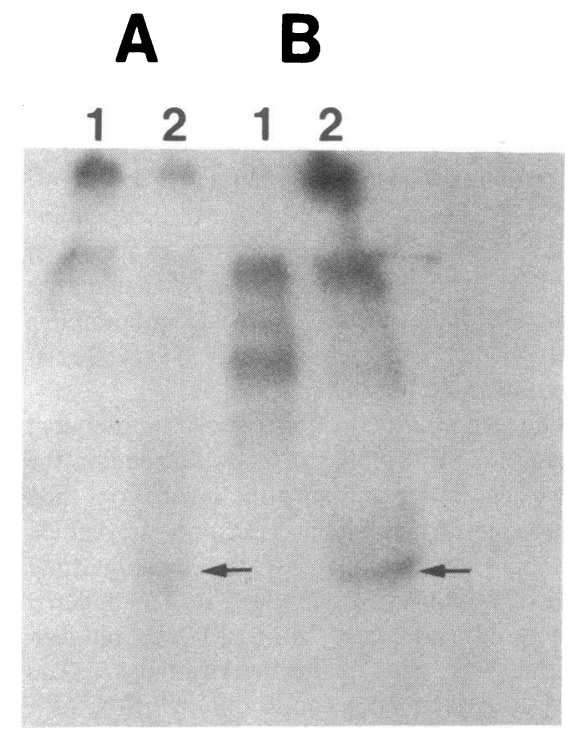

Figure 3. Radioautogram of polyacrylamide slab gel electrophoresis. The washed clot prepared from purified fibrinogen $(A)$ or from normal plasma $(B)$ was suspended in buffered saline containing XIIIa (0.4 $\mathrm{U} / \mathrm{ml}$ ) and incubated at $37^{\circ} \mathrm{C}$ for $4 \mathrm{~h}$. The washed clot together with the suspending buffered saline, as a whole, was lyophilized, solubilized, and subjected to electrophoresis on a slab gel before (lane 1) or after (lane 2) the incubation. Arrows indicate free $\alpha_{2}$ PI. For details, see Methods. 


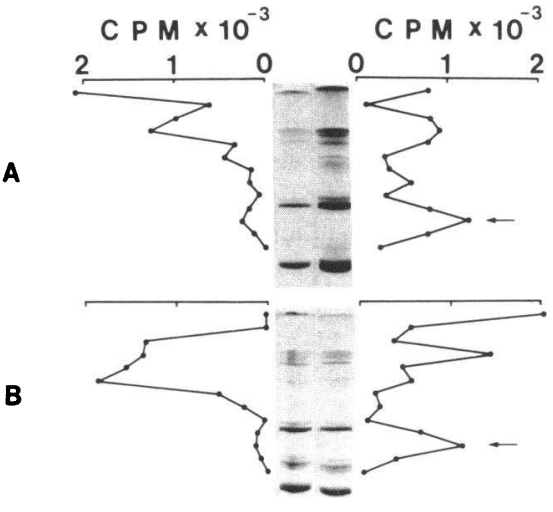

Figure 4. Distribution of radiolabeled $\alpha_{2} \mathrm{PI}$ in polyacrylamide disc gel electrophoresis. The washed clot prepared from purified fibrinogen (upper panel $A$ ) or from normal plasma (lower panel $B$ ) was suspended and incubated in buffered saline as in Fig. 3. The washed clot together with the suspending buffered saline, as a whole, was lyophilized, solubilized, and subjected to disc gel electrophoresis before (left) or after (right) the incubation. After electrophoresis, the disc gel was cut transversely into slices, and each slice was counted for radioactivity. Arrows indicate free $\alpha_{2}$ PI corresponding to relative molecular weight $\simeq$ 70,000 .

the incorporation of $\alpha_{2} \mathrm{PI}$ were then measured. The incorporation as well as the release was negligibly small in the control experiments in which EDTA and iodoacetamide were added. The net release of $\alpha_{2}$ PI from the washed clot into the suspending plasma was calculated by subtracting the amount of incorporated $\alpha_{2} \mathrm{PI}$ from the amount of released $\alpha_{2}$ PI. The results shown in Fig. 6 indicate that the net release of $\alpha_{2} \mathrm{PI}$ from the clot was decreased when the concentration of $\alpha_{2} \mathrm{PI}$ in the suspending plasma was increased. It also indicates that the net release was nearly zero when the concentration of $\alpha_{2} \mathrm{PI}$ in the suspending plasma was the same as that of the plasma from which the clot had been prepared.

Acceleration of $\alpha_{2} P I$ release by $N$-peptide. The washed fibrin clot was prepared as described above from normal plasma containing radiolabeled $\alpha_{2} \mathrm{PI}$. The clot was suspended and incubated in normal heparinized plasma with or without the presence of N-peptide.

As seen in Fig. 7, radiolabeled $\alpha_{2}$ PI was gradually released from the fibrin clot on incubation. Here again, the release was suppressed by the presence of EDTA and iodoacetamide which

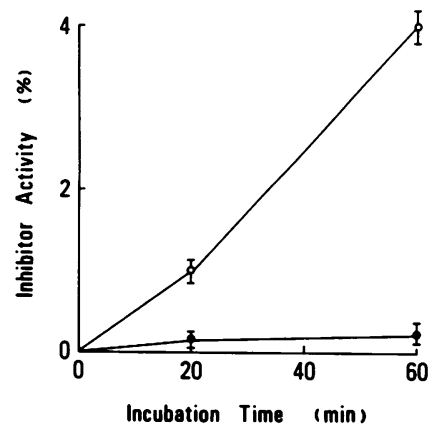

Figure 5. Release of the inhibitor activity. The washed fibrin clot prepared from normal plasma was suspended and incubated at $37^{\circ} \mathrm{C}$ in buffered saline containing XIIIa $(0.6$ $\mathrm{U} / \mathrm{ml})(0)$. As a control, EDTA and iodoacetamide were added to the suspending buffered saline (•). The total immediatetype antiplasmin activity released into the suspending buffered saline was measured and

expressed as a percent of the immediate-type antiplasmin activity present in the original whole plasma.

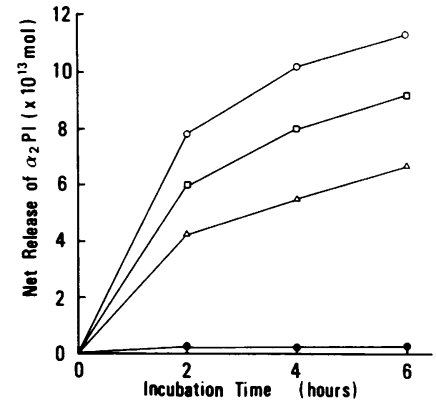

Figure 6. Net release of $\alpha_{2} \mathrm{PI}$ from fibrin clots suspended in plasma with various concentrations of $\alpha_{2}$ PI. The washed fibrin clot prepared from $100 \mu \mathrm{l}$ of normal citrated plasma was suspended in $500 \mu \mathrm{l}$ of $\alpha_{2}$ PI-deficient plasma (0), a 3:1 mixture of $\alpha_{2}$ PI-deficient plasma and normal plasma $\left(\square, \alpha_{2} \mathrm{PI} 0.2\right.$ $\mu \mathrm{M})$, a 1:1 mixture of $\alpha_{2}$ PI-deficient plasma and normal

plasma $\left(\Delta, \alpha_{2}\right.$ PI $\left.0.4 \mu \mathrm{M}\right)$, and normal plasma $\left(\bullet, \alpha_{2}\right.$ PI $\left.0.8 \mu \mathrm{M}\right)$. The suspending plasmas were heparinized ones and contained aprotinin. For the measurement of the release, the normal plasma containing a trace amount of radiolabeled $\alpha_{2}$ PI was used for preparation of the washed clot. For the measurement of the incorporation, the normal plasma containing a fixed amount of radiolabeled $\alpha_{2}$ PI was used for preparation of the suspending plasma milieus. The net release was calculated by subtracting the value of the incorporation from the value of the release. For details, see Methods.

inhibit XIIIa. The release was increased by the presence of $\mathrm{N}$ peptide (Fig. 7), and the increase was dependent on the concentrations of N-peptide (Fig. 8).

Acceleration of fibrinolysis by the release of $\alpha_{2} P I$ from fibrin clot. Normal plasma containing radiolabeled fibrinogen and exogenously added t-PA was clotted by adding thrombin and calcium chloride. After incubation for $30 \mathrm{~min}$ at $37^{\circ} \mathrm{C}$ the clot formed was squeezed and washed to remove unbound materials. The clot retained the t-PA adsorbed onto fibrin. The clot was then suspended and incubated in buffered saline containing plasminogen. The suspending buffered saline additionally contained XIIIa or EDTA/iodoacetamide. Fibrinolysis was followed by counting of the radioactivity released into the suspending buffered saline at time intervals.

As seen in Fig. 9, fibrinolysis was accelerated when XIIIa was present in the suspending buffered saline as compared with the fibrinolysis observed in the presence of EDTA/iodoacetamide. The release of $\alpha_{2} \mathrm{PI}$ in these experiments was measured using radiolabeled $\alpha_{2} \mathrm{PI}$ instead of radiolabeled fibrinogen, as in Fig. 2, in which fibrinolysis was suppressed by aprotinin. Approximately $20 \%$ of the $\alpha_{2}$ PI bound to fibrin was released after $2 \mathrm{~h}$ of incubation in the presence of exogenously added XIIIa,

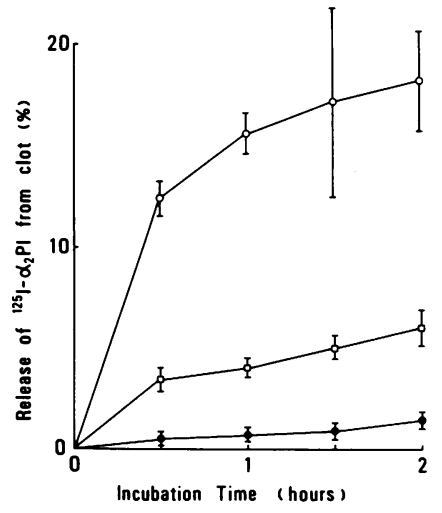

Figure 7. Release of $\alpha_{2}$ PI from fibrin clots on incubation by $\mathrm{N}$ peptide. The washed fibrin clot prepared from normal plasma containing radiolabled $\alpha_{2} \mathrm{PI}$ was suspended and incubated at $37^{\circ} \mathrm{C}$ in normal heparinized plasma containing aprotinin and no (口) or $500 \mu \mathrm{M}(0) \mathrm{N}$ peptide. As a control, EDTA and iodoacetamide were added to the suspending plasma (•). After various lengths of time, cumulative release of radiolabel from the clot into the suspending medium was measured and expressed as a percentage of the total radioactivity, as in Fig. 1. 


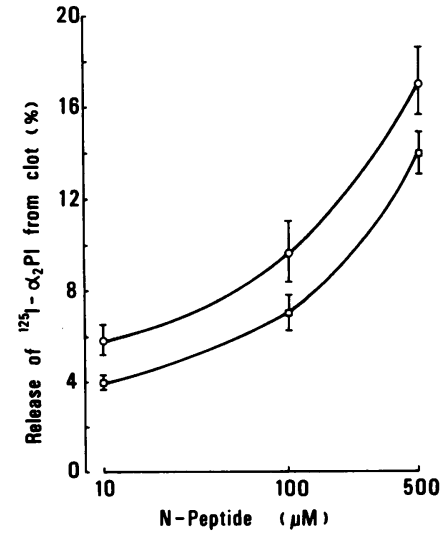

The release in the presence of EDTA/iodoacetamide was subtract The abscissa is on a logarithmic scale.

whereas the release was only $<1 \%$ in the presence of EDTA/ iodoacetamide which inhibited XIIIa endogenously generated in the clot.

When an $\alpha_{2}$ PI-deficient plasma clot was used instead of a normal plasma clot in the experiments of Fig. 9, the lysis was very rapid and completed in less than $1 \mathrm{~h}$. Furthermore, there was no difference of fibrinolysis whether EDTA/iodoacetamide was added or not, in contrast to a significant difference observed with the normal plasma clot in Fig 9. This indicates that EDTA/ iodoacetamide did not influence the fibrinolytic process itself, at least under the experimental conditions used.

Normal platelet-rich plasma containing radiolabeled fibrinogen was clotted by the addition of calcium chloride. After 30 min of incubation at $37^{\circ} \mathrm{C}$, the retracted clot was suspended in normal plasma containing a fixed amount of exogenously added $\mathrm{t}$-PA and various concentrations of $\mathrm{N}$-peptide and was then further incubated at $37^{\circ} \mathrm{C}$. Fibrinolysis was followed by counting of the radioactivity released into the suspending plasma at time intervals.

As seen in Fig. 10, fibrinolysis was accelerated by the presence of $\mathrm{N}$-peptide, and the acceleration was dependent on the concentrations of $\mathrm{N}$-peptide. The rate of fibrinolysis was nearly proportional to the degree of the release of $\alpha_{2}$ PI achieved by $\mathrm{N}$ peptide at the end of $2 \mathrm{~h}$ of incubation (Fig. 11). The extent of

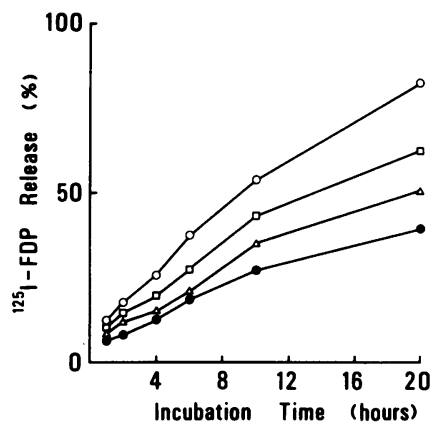

Figure 10. Acceleration of fibrinolysis by the release of $\alpha_{2} \mathrm{PI}$ caused by N-peptide. Plateletrich normal plasma containing radiolabeled fibrinogen was clotted by the addition of calcium ions. After 30-min incubation at $37^{\circ} \mathrm{C}$, the clot formed was suspended and incubated in platelet-poor plasma containing a fixed amount of t-PA, hirudin, and various concentrations of N-peptide. The con-

centrations of $\mathrm{N}$-peptide were $500(0), 100(\square)$, and $10 \mu \mathrm{M}(\Delta)$. Control without $\mathrm{N}$-peptide (๑). After various lengths of incubation, cumulative release of radiolabel from the clot into the suspending plasma was measured and expressed as a percentage of the total radioactivity, as in Fig. 9. Each point represents the average of three measurements.

release at the end of $2 \mathrm{~h}$ of incubation represents nearly the maximum release achieved by each concentration of $\mathrm{N}$-peptide, since the cumulative release reached a plateau at around $2 \mathrm{~h}$ of incubation. This indicates that the maximum extent of release which was achieved during the initial $2 \mathrm{~h}$ of incubation determined the rate of the following fibrinolytic process seen in Fig. 10. When $\alpha_{2}$ PI-deficient plasma or XIII-deficient plasma was used instead of normal plasma in similar experiments, no acceleration of fibrinolysis was observed in the presence of $\mathrm{N}$-peptide (Fig. 12).

\section{Discussion}

$\alpha_{2} \mathrm{PI}$ is cross-linked to the fibrin $\alpha$-chain when blood coagulation takes place (2-4). The reaction proceeds rapidly to reach a maximum and then levels off, with only around $20 \%$ of the $\alpha_{2} \mathrm{PI}$ being cross-linked (2). We have suggested that this self-limiting nature of the cross-linking reaction may be due to the reaction equilibrium favoring dissociation of the cross-linked complex rather than the development of structural hindrance in the polymerizing fibrin(ogen) (9). The concept is mainly based on the observation that the $\alpha_{2} \mathrm{PI}$-fibrinogen cross-linking reaction is a reversible one, and the isolated complex of $\alpha_{2} \mathrm{PI}$ and fibrinogen could be dissociated rapidly into each of its components by XIIIa

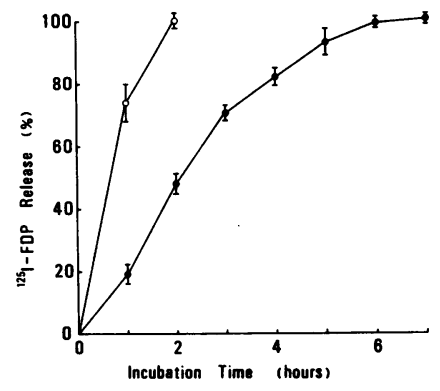

Figure 9. Acceleration of fibrinolysis by the release of $\alpha_{2} \mathrm{PI}$. The washed fibrin clot prepared from normal plasma containing radiolabeled fibrinogen and t-PA $(5.5 \mathrm{U} / \mathrm{ml})$ was suspended and incubated at $37^{\circ} \mathrm{C}$ in buffered saline containing Glu-plasminogen and XIIIa $(1.8 \mathrm{U} / \mathrm{ml})$ (o) or in buffered saline containing Glu-plasminogen, EDTA, and iodoacetamide (๑). After various lengths of incubation, cumulative release of radiolabel from the clot into the suspending buffered saline was measured and expressed as a percentage of the total radioactivity. Data represent the mean $\pm \mathrm{SD}$ of triplicate experiments. For details see Methods.

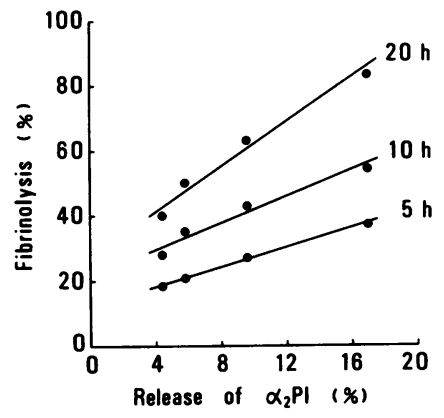

Figure 11. Linear relationship between the release of $\alpha_{2}$ PI caused by $\mathrm{N}$-peptide and the rate of fibrinolysis. The percent values of release of $\alpha_{2}$ PI from the fibrin clot caused by N-peptide was adopted from the $2 \mathrm{~h}$ values in Figs. 7 and 8, which were nearly maximum, and plateau values achieved by each concentration of $\mathrm{N}$-peptide. The extents of fibrinolysis in the presence of various concentrations of N-peptide, corresponding to those in Fig. 8, are those achieved at the incubation times indicated at the end of each line and adopted from the data in Fig. 10. 


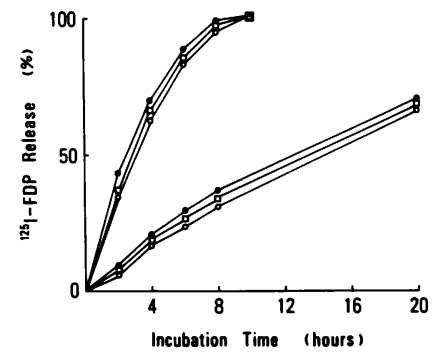

Figure 12. Influence of N-peptide on fibrinolysis of $\alpha_{2} \mathrm{PI}$ - or Factor XIII-deficient plasma. Platelet-rich $\alpha_{2}$ PI-deficient plasma (upper portion) or Factor XIII-deficient plasma (lower portion) containing radiolabeled fibrinogen was clotted, suspended, and incubated in platelet-poor $\alpha_{2} \mathrm{PI}$ - or Factor XIII-deficient plasma containing various concentrations of $\mathrm{N}$-peptide, respectively. The experimental conditions were the same as those in Fig. 10, except for the omission of t-PA in $\alpha_{2}$ PI-deficient plasma. The concentrations of N-peptide were $500(0)$ and $100 \mu \mathrm{M}(\square)$. Control without N-peptide (๑). After various lengths of incubation, cumulative release of radiolabel from the clot into the suspending plasma' was measured and expressed, as in Fig. 10.

(9). In the present study, we have extended our investigation further to see if the $\alpha_{2}$ PI-fibrin cross-linking reaction is also a reversible one. The question is crucial because $\alpha_{2} \mathrm{PI}$ may be mainly cross-linked to fibrin and not much with fibrinogen when a thrombus is formed in vivo, and dissociation of the $\alpha_{2}$ PI-fibrin cross-linked complex would be of great consequence in thrombolysis.

When the fibrin clot prepared from normal plasma was suspended and incubated in $\alpha_{2}$ PI-deficient plasma or buffered saline, $\alpha_{2}$ PI which had been cross-linked to fibrin was gradually released from the fibrin clot (Fig. 1). The release was most likely mediated by XIIIa since the rate of the release was dependent on the amount of XIIIa present (Fig. 2) and the release was suppressed by the presence of EDTA and iodoacetamide (Fig. 1) which are known to inhibit XIIIa $(25,26)$. The release progressed very slowly and took hours to level off (Fig. 1). This slow progress of the release is in contrast to the very rapid dissociation of the $\alpha_{2}$ PI-fibrinogen complex which was completed in only a few minutes (9). The difference may be explained by the structural tightness of the cross-linked fibrin clot which hinders the enzyme (XIIIa) from gaining access to the substrate $\alpha_{2}$ PI-fibrin complex. The release slowed down after $2 \mathrm{~h}$ of incubation, and every effort to accelerate the release, including frequent changes of the suspending media and replenishment of fresh XIIIa, was unsuccessful. We speculate that the highly polymerized $\alpha$-chain of fibrin formed after $2 \mathrm{~h}$ of incubation may have hindered the release. We have previously shown that the $\alpha_{2}$ PI- $\alpha$-chain monomer complex formed at the initial stage of clotting was gradually transformed to the $\alpha_{2}$ PI- $\alpha$-chain polymer complex as the $\alpha$ chain cross-linking polymerization proceeded $(3,9)$. This was also seen in the present study (Fig. $4 \mathrm{~B}$ ). The transformation was completed after $2 \mathrm{~h}$ of incubation and no $\alpha_{2} \mathrm{PI}-\alpha$-chain monomer complex was left (3). Probably XIIIa could gain access to the $\alpha_{2}$ PI cross-linked to $\alpha$-chain monomer, but not to the $\alpha_{2}$ PI cross-linked to $\alpha$-chain polymer.

When $\alpha_{2} \mathrm{PI}$ was present in the suspending medium, some of the $\alpha_{2}$ PI was incorporated by XIIIa into the clot in exchange for the release of fibrin-bound $\alpha_{2} \mathrm{PI}$ (replacement) and the incorporation was proportional to the concentration of $\alpha_{2} \mathrm{PI}$ in the suspending medium. Therefore, the net release of $\alpha_{2} \mathrm{PI}$ from the suspended clot was inversely related to the concentrations of free $\alpha_{2}$ PI in the suspending medium (Fig. 6). When the concentration of $\alpha_{2} \mathrm{PI}$ in the suspending medium was the same as that of the original plasma from which the clot had been prepared, the net release was nearly zero, since the incorporation and the release were balanced (Fig. 6).

XIIIa is classified as a transglutaminase and is also called plasma transglutaminase (27). The findings in the present and previous studies that $\alpha_{2}$ PI could be released from the $\alpha_{2}$ PI-fibrin(ogen) complex by XIIIa are in accordance with and fully expected from the general reaction mechanism for transglutaminases proposed by Folk (7) and Chung and Folk (8) and reviewed by Lorand and Conrad (28).

$\mathrm{N}$-peptide, which contains the residue involved in the crosslinking reaction with fibrin(ogen), was shown to be rapidly crosslinked with fibrin, and competitively inhibits the cross-linking of $\alpha_{2} \mathrm{PI}$ with fibrin when it is present concurrently with $\alpha_{2} \mathrm{PI}$ at the time of clotting in a purified system (29) as well as in plasma (30). When the preformed fibrin clot containing radioactive $\alpha_{2} \mathrm{PI}$ cross-linked with fibrin was suspended in normal plasma, the fibrin-bound $\alpha_{2}$ PI was replaced by free $\alpha_{2}$ PI present in the suspending plasma, and radioactive $\alpha_{2} \mathrm{PI}$ was gradually released from the fibrin clot (Fig. 7). This replacement of the bound $\alpha_{2} \mathrm{PI}$ was most likely mediated by XIIIa because EDTA and iodoacetamide inhibit the release (Fig. 7). When the N-peptide was present in the suspending plasma, further replacement of the bound $\alpha_{2}$ PI by N-peptide occurred, and the release was increased (Fig. 7). The increase was dependent on the concentrations of N-peptide (Fig. 8), which was incorporated into the clot in exchange for $\alpha_{2} \mathrm{PI}$.

In the previous studies, $\alpha_{2} \mathrm{PI}$ cross-linked with fibrin was shown to play a significant role in inhibition of physiologically occurring fibrinolysis, thus stabilizing thrombi, including hemostatic plugs (6), although the amount of $\alpha_{2}$ PI cross-linked to fibrin is very small and only $1 \mathrm{~mol} / 20 \mathrm{~mol}$ of fibrin (monomer). The importance of cross-linking of $\alpha_{2}$ PI with fibrin was further supported by the previous finding that the presence of $\mathrm{N}$-peptide in plasma at the time of clotting accelerated the subsequent fibrinolytic process by inhibiting the cross-linking of $\alpha_{2}$ PI (30) and the extent of fibrinolysis was found to be proportional to the degree of inhibition of $\alpha_{2}$ PI cross-linking (30). In the present study, it was further demonstrated that the release of $\alpha_{2}$ PI from the fibrin clot results in an acceleration of fibrinolysis.

Normal plasma supplemented with t-PA was clotted in the presence of calcium ions and incubated at $37^{\circ} \mathrm{C}$ for $30 \mathrm{~min}$, during which the cross-linking of $\alpha_{2}$ PI with fibrin was completed, but no significant fibrinolysis took place. The fibrin clot thus prepared was suspended in buffered saline containing plasminogen. When the suspending buffered saline contained XIIIa in addition and the release of $\alpha_{2}$ PI from the fibrin clot was accelerated, as seen in Fig. 2, the fibrinolysis was significantly accelerated (Fig. 9) as compared with the control where XIIIa was suppressed be EDTA/iodoacetamide and the release of $\alpha_{2} \mathrm{PI}$ was negligibly small (Fig. 2).

The retracted plasma clot prepared from normal plateletrich plasma in the presence of calcium ions was suspended in normal plasma containing a fixed amount of exogenously added $\mathrm{t}-\mathrm{PA}$ and various concentrations of $\mathrm{N}$-peptide. The presence of $\mathrm{N}$-peptide in the suspending plasma accelerated fibrinolysis (Fig. 10 ), and the rate of fibrinolysis was proportional to the amount of the $\alpha_{2}$ PI released (replaced) by N-peptide (Fig. 11). The acceleration was not seen when $\alpha_{2}$ PI-deficient or XIIIa-deficient plasma was used (Fig. 12), indicating that the acceleration was caused by the XIIIa-catalyzed release of $\alpha_{2}$ PI from fibrin. When 
the nonretracted plasma clot prepared from platelet-poor plasma was suspended in plasma containing $\mathrm{N}$-peptide, the acceleration caused by $\mathrm{N}$-peptide was not so remarkable as that seen with the retracted plasma clot. As has been suggested in the previous study (6), inhibition of fibrinolysis of a retracted plasma clot may be more dependent on $\alpha_{2}$ PI bound to fibrin as compared with a nonretracted plasma clot, and the reduction of the amount of fibrin-bound $\alpha_{2}$ PI by N-peptide may have produced a more pronounced effect on the fibrinolysis of the retracted clot than on the fibrinolysis of the nonretracted clot.

All the findings presented here may be extrapolated to in vivo situations. Under thrombolytic therapy with urokinase or streptokinase, $\alpha_{2} \mathrm{PI}$ is consumed by forming a complex with generated plasmin and being removed from the circulation (19, $31)$. Consequent reduction of $\alpha_{2}$ PI level in the circulation has been considered to be unfavorable because it may induce a generalized hemorrhagic tendency. However, the reduction of $\alpha_{2} \mathrm{PI}$ level might also bring about some beneficial effects in view of thrombolysis (32) because the reduction of $\alpha_{2}$ PI level would facilitate the release of $\alpha_{2}$ PI from thrombi as seen in Figs. 1 and 6 , when thrombi are fresh and fibrins are not highly cross-linked, thus accelerating spontaneously occurring fibrinolysis. This is likely to occur since more XIIIa may be continuously generated on the surface of thrombi in in vivo situations as compared with the in vitro situations employed in the present study where XIIIa generation was limited. In fact, Kumada and Abiko demonstrated that reduction of $\alpha_{2}$ PI level in the circulation induced spontaneous thrombolysis in animal experiments (33). They experimentally produced thrombi in rats. After development of thrombi, the $\alpha_{2}$ PI level in the circulation was reduced and maintained at $<50 \%$ of normal by repeated injections of anti- $\alpha_{2} \mathrm{PI}$ $\mathrm{F}\left(\mathrm{ab}^{\prime}\right)_{2}$. The average size of thrombi of the rats whose $\alpha_{2}$ PI level had been reduced was significantly smaller than that of the controls and approximately half of the control values. Moreover, the average size of thrombi was significantly smaller than the initial size of thrombi observed at the start of treatment with anti- $\alpha_{2} \mathrm{PI}$; the average size was approximately two-thirds of the initial size. Significantly higher levels of FDP were observed in the anti- $\alpha_{2}$ PI treated group. These findings indicated that reduction of $\alpha_{2}$ PI level in the circulation induced spontaneous thrombolysis, thereby not only preventing the growth of thrombi but also accelerating the dissolution of thrombi already formed. Probably the reduction of $\alpha_{2}$ PI level induced the release of $\alpha_{2}$ PI from thrombi and facilitated the spontaneously occurring fibrinolysis.

The results of the present study may imply that the rapid and sustained reduction of $\alpha_{2}$ PI level in the circulation by pharmacological or any other means, if possible, would cause the release of $\alpha_{2}$ PI from thrombi and facilitate thrombolysis induced by the physiologically occurring fibrinolytic process or exogenously administered plasminogen activators.

\section{Acknowledgments}

We would like to thank Ms. M. Takano for her help in preparation of the manuscript.

This study was supported in part by a research grant from the Ministry of Education, Japan.

\section{References}

1. Aoki, N., and P. C. Harpel. 1984. Inhibitors of the fibrinolytic enzyme system. Semin. Thromb. Hemostasis. 10:24-41.
2. Sakata, Y., and N. Aoki. 1980. Cross-linking of $\alpha_{2}$-plasmin inhibitor to fibrin by fibrin-stabilizing factor. J. Clin. Invest. 65:290-297.

3. Tamaki, T., and N. Aoki. 1981. Cross-linking of $\alpha_{2}$-plasmin inhibitor and fibronectin to fibrin by fibrin-stabilizing factor. Biochim. Biophys. Acta. 661:280-286.

4. Tamaki, T., and N. Aoki. 1982. Cross-linking of $\alpha_{2}$-plasmin inhibitor to fibrin catalyzed by activated fibrin-stabilizing factor. J. Biol. Chem. 257:14767-14772.

5. Aoki, N., Y. Sakata, and A. Ichinose. 1983. Fibrin-associated plasminogen activation in $\alpha_{2}$-plasmin inhibitor deficiency. Blood. 62:11181122.

6. Sakata, Y., and N. Aoki. 1982. Significance of cross-linking of $\alpha_{2}-$ plasmin inhibitor to fibrin in inhibition of fibrinolysis and in hemostasis. J. Clin. Invest. 69:536-542.

7. Folk, J. E. 1969. Mechanism of action of guinea pig liver transglutaminase. VI. Order of substrate addition. J. Biol. Chem. 244:37073713.

8. Chung, S. I., and J. E. Folk. 1972. Kinetic studies with transglutaminases. J. Biol. Chem. 247:2798-2807.

9. Ichinose, A., and N. Aoki. 1982. Reversible cross-linking of $\alpha_{2-}$ plasmin inhibitor to fibrinogen by fibrin-stabilizing factor. Biochim. Biophys. Acta. 706:158-164.

10. Aoki, N., H. Saito, T. Kamiya, K. Koie, Y. Sakata, and M. Kohakura. 1979. Congenital deficiency of $\alpha_{2}$-plasmin inhibitor associated with severe hemorrhagic tendency. J. Clin. Invest. 63:877-884.

11. Moroi, M., and N. Aoki. 1976. Isolation and characterization of $\alpha_{2}$-plasmin inhibitor from human plasma. J. Biol. Chem. 251:59565965.

12. Blombäck, B., and M. Blombäck. 1956. Purification of human and bovine fibrinogen. Ark. Kemi. 10:415-443.

13. Matsuda, M., S. Iwanaga, and S. Nakamura. 1972. A simple, large scale method for preparation of plasminogen-free fibrinogen. Thromb. Res. 1:619-630.

14. Vuento, M., and A. Vaheri. 1978. Dissociation of fibronectin from gelatin-agarose by amino compounds. Biochem. J. 175:333-337.

15. Curtis, C. G., and L. Lorand. 1976. Fibrin-stabilizing factor (Factor XIII). Methods Enzymol. 45:177-191.

16. Lorand, L., O. M. Lockridge, L. K. Campbell, R. Myhrman, and J. Bruner-Lorand. 1971. Transamidating enzymes II. A continuous fluorescent method suited for automating measurements of factor XIII in plasma. Anal. Biochem. 44:221-231.

17. Lundblad, R. L. 1971. A rapid method for the purification of bovine thrombin and the inhibition of the purified enzyme with phenyl methyl sulfonyl fluoride. Biochemistry. 10:2501-2505.

18. Naito, K., and N. Aoki. 1977. Assay of $\alpha_{2}$-plasmin inhibitor activity by means of a plasmin specific tripeptide substrate. Thromb. Res. 12:1147-1156.

19. Aoki, N., M. Moroi, M. Matsuda, and K. Tachiya. 1977. The behavior of $\alpha_{2}$-plasmin inhibitor in fibrinolytic states. J. Clin. Invest. 60: 361-369.

20. Rijken, D. C., and D. Collen. 1981. Purification and characterization of the plasminogen activator secreted by human melanoma cells in culture. J. Biol. Chem. 256:7035-7041.

21. Wallen, P., and B. Wiman. 1972. Characterization of human plasminogen. Separation and partial characterization of different molecular forms of human plasminogen. Biochim. Biophys. Acta. 257:122134.

22. Mattler, L. E., and N. U. Bang. 1977. Serine protease specificity for peptide chromogenic substrates. Thromb. Hemostasis. 38:776-792.

23. Laemmli, U. K. 1970. Cleavage of structural proteins during the assembly of the head of bacteriophage T4. Nature (Lond.). 227:680-685.

24. Folk, J. E., and S. I. Chung. 1975. Blood coagulation factor XIII: relationship of some biological properties to subunit structure. In Proteases and Biological Control. E. Reich, D. B. Rifkin, and E. Shaw, editors. Cold Spring Harbor Laboratories, Cold Spring Harbor, NY. 157-170.

25. Curtis, C. G., P. Stenberg, C.-H. J. Chou, A. Gray, K. L. Brown, 
and L. Lorand. 1973. Titration and subunit localization of active center cysteine in fibrinoligase (thrombin-activated fibrin stabilizing factor). Biochem. Biophys. Res. Commun. 52:51-56.

26. Chung, S. I., M. S. Lewis, and J. E. Folk. 1974. Relationship of the catalytic properties of human plasma and platelet transglutaminases (activated blood coagulation factor XIII) to their subunit structures. $J$. Biol. Chem. 249:940-950.

27. Folk, J. E., and J. S. Finlayson. 1977. The $\epsilon-(\gamma$-glutamyl) lysine crosslink and the catalytic role of transglutaminases. Adv. Protein. Chem. 31:1-133.

28. Lorand, L., and S. M. Conrad. 1984. Transglutaminases. Mol. Cell. Biochem. 58:9-35.

29. Ichinose, A., T. Tamaki, and N. Aoki. 1983. Factor XIII-mediated cross-linking of $\mathrm{NH}_{2}$-terminal peptide of $\alpha_{2}$-plasmin inhibitor to fibrin. FEBS (Fed. Eur. Biochem. Soc.) Lett. 153:369-371.

30. Kimura, S., T. Tamaki, and N. Aoki. 1985. Acceleration of fibrinolysis by $\mathrm{N}$-terminal peptide of $\alpha_{2}$-plasmin inhibitor. Blood. 66:157160.

31. Collen, D., and M. Verstraete. 1979. $\alpha_{2}$-Antiplasmin consumption and fibrinogen breakdown during thrombolytic therapy. Thromb. Res. 14:631-739.

32. Hayashi, S., and Yamada, K. 1979. Role of $\alpha_{2}$-plasmin inhibitor in the appearance of fibrinolytic activity during urokinase administration, and an evaluation of the optimal urokinase dosage. Thromb. Res. 16: 393-400.

33. Kumada, T., and Y. Abiko. 1984. Physiological role of $\alpha_{2}$-plasmin inhibitor in rats. Thromb. Res. 36:153-163. 